\title{
Rheology and Thermal Studies of Stearoyl Chitosan Varying the Degree of Substitution
}

\author{
Pushpika Katugampola ${ }^{1}$, Cherese Winstead Casson ${ }^{2 *}$ \\ ${ }^{1,2}$ Department of Chemistry, Delaware State University, Dover, DE 19901, U.S.A.
}

\begin{abstract}
Two compounds of stearoyl chitosan; degree of substitution (DS) 79\% and DS 86\%, were synthesized by using the starting material as chitosan and deacetylated chitosan respectively. Fourier transform infrared (FT-IR) and Nuclear Magnetic Resonance $\left({ }^{1} H\right.$ NMR) spectroscopy were used to confirm the chemical structure of prepared products. The thermal stability of stearoyl chitosan decreased with increased DS while the DO (degree of order) increased with increased DS. The 2\% (w/v) stearoyl chitosan (DS 86\%) solutions displayed shear thinning behavior. Also, the shear stress and viscosity values were higher for stearoyl chitosan dissolved in pyridine than in toluene at the same shear rate. Since $2 \%$ stearoyl chitosan dissolved in pyridine had high viscosity with shear thinning property, it can be suitable as rheology modifiers.
\end{abstract}

Keywords: acylation, deacetylation, rheology, stearoyl chitosan, thermal stability

\section{Introduction}

Chitosan, cationic copolymer of D-glucosamine and $\mathrm{N}$-acetylglucosamine, is a natural linear biopolyaminosachcharide. It has an amphiphilic character due to the presence of hydrophilic amino groups and hydrophobic acetamide groups in its molecular structure. Due to its non-toxicity, biodegradability, biocompatibility, low immunogenicity, antimicrobial activity, physiochemical, and biological properties, it can be used in various fields. But the poor solubility in water and most common organic solvents limits its applications [1,2].

One of the chemical modification methods that increase organic solubility of chitosan is acylation. Stearoyl chitosan is one of the derivatives of acylated chitosan. The substitution reaction between stearoyl groups and hydrogen atoms of both hydroxyl and amino groups of chitosan results O-stearoyl chitosan and N-stearoyl chitosan, respectively (Scheme 1). But the main focus of this study was on $\mathrm{N}$-stearoyl chitosan, which was the major product.

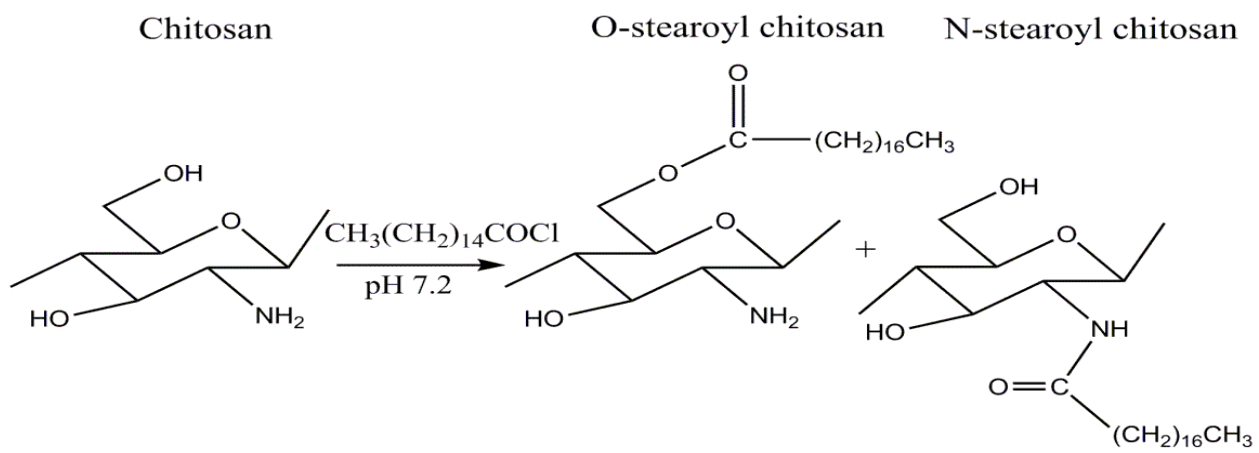

Scheme 1. The synthetic route for stearoyl chitosan.

Stearoyl chloride, which has C18; was selected for chitosan acylation due to its long hydrocarbon chain, which can lead to increase hydrophobicity of stearoyl chitosan. Thereby increase its organic solubility. By increasing the organic solubility results high viscous solutions. According to solubility studies, steaoryl chitosan can be dissolved in hexafluoroacetone [3].

The removal of acetyl groups from the molecular chain of chitin and leaving behind amino groups $\left(-\mathrm{NH}_{2}\right)$ that determines the reactivity of chitosan is known as the process of deacetylation (Scheme 1.1). DD (degree of deacetylation) is defined as the molar fraction of D-glucosamine residues in the chitosan chain $[4,5]$. The quality and physicochemical characteristics of chitosan vary with DD. The DD value is used to differentiate chitin from chitosan, since the DD value of $75 \%$ or above is known as chitosan [6]. There are methods available to deacetylate chitin/chitosan. For example, using higher temperature or increasing the strength of $\mathrm{NaOH}$ solution can enhance the removal of acetyl groups from chitin/chitosan polymer. An example for deacetylation of chitosan is starting with DD 75\% chitosan and ends up with DD 99.8\% chitosan [7]. 


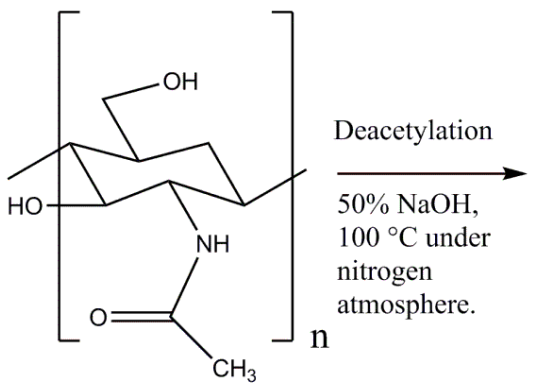

Chitin

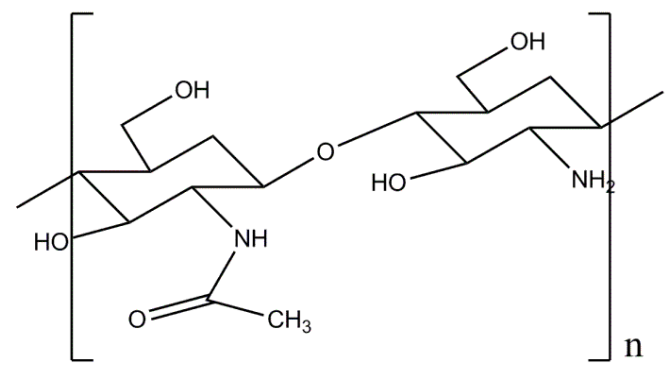

Chitosan

Scheme 2. The process of deacetylation.

Rheology, the science of deformation and flow of matter, investigates the response of materials to an applied stress or strain. There are two types of rheological behavioral patterns: viscous behavior and elastic behavior. The rheological behavior of polymer solutions can be explained using equation (1.1), which is the power law equation [8-11].

$$
\tau=A \dot{\gamma}^{n}
$$

where A is flow consistency index, which is derived from the curve logarithm shear stress versus logarithm shear rate and $\mathrm{n}$ is flow behavior index. $\tau$ is shear stress, $\dot{\gamma}$ is shear rate and $\eta$ is shear viscosity. For a Newtonian fluid, $\mathrm{n}=1$ and $\mathrm{A}=\eta$. Most of the polymer solutions show non-Newtonian characteristics. Therefore, polymers exhibit shear thinning at higher shear rates. When the shear rate increases, disruptions occur faster than the chains can reentangle and the resultant decreasing entanglement allows molecules to flow with less resistance, which leads to decrease in viscosity [8-11].

This paper describes the preparation of two different degree substituted stearoyl chitosan (DS 79\% and DS $86 \%$ ) by using the starting material as chitosan and deacetylated chitosan respectively. Spectroscopy methods such as FT-IR, and ${ }^{1} \mathrm{H}$ NMR were used to confirm the chemical structure of the palmitoyl chitosan substituents. TGA studies were carried out to determine the thermal stability while XRD analysis was used to characterize the crystalline structure of palmitoyl chitosan. The rheological behavior of the solutions of stearoyl chitosan with two different degree substituents was assessed to investigate its' applicability in various fields such as fabricating nanofibers by electrospinning process, using as viscosity inducing agents in pharmaceutical formulations, and as rheology modifiers in industrial applications.

\subsection{Materials}

\section{Materials and Methods}

Chitosan (MMW, Degree of deacetylation 75-85\%) was obtained from Sigma-Aldrich Co. (St. Louis, MO, USA). Monochloroacetic acid was purchased from Fisher Chemical Co. (NJ, USA). Hexanoyl chloride, palmitoyl chloride, stearoyl chloride, deuterated solvents (d-acetic acid, d-trifluoroacetic acid and deuterium oxide) and other chemicals used in this study were purchased from Fisher Scientific (PA, USA). All chemicals used were ACS grade.

\subsection{Preparation of deacetylated chitosan with a DD value of $94 \%$}

Deacetylated chitosan was synthesized by 'sodium hydroxide deacetylation of chitosan [12-14]. Chitosan, which has a DD of $75-85 \%(5 \mathrm{~g})$, was reacted with $50 \%$ sodium hydroxide solution $(50 \mathrm{~mL})$ at a $1: 10(\mathrm{~W} / \mathrm{V})$ ratio in a round bottom flask $(250 \mathrm{~mL})$ under nitrogen atmosphere for four hours at $100{ }^{\circ} \mathrm{C}$. The filtered, neutral product was dried in an oven (Isotemp Oven, Fisher Scientific, PA, USA) for 24 hours at $40{ }^{\circ} \mathrm{C}$, then in a vacuum oven (Isotemp Model 280A, Fisher Scientific, PA, USA) for 24 hours at $40{ }^{\circ} \mathrm{C}$.

The DD of chitosan was determined using $\mathrm{pH}$-metric titration. According to the procedure, the dried chitosan $(0.2 \mathrm{~g})$ was dissolved in a mixture of $20 \mathrm{~mL} 0.1 \mathrm{M}$ hydrochloric acid and $25 \mathrm{~mL}$ deionized water [5]. After 30 minutes of continuous stirring, $25 \mathrm{~mL}$ of deionized water added and stirring was continued for another 30 minutes. Finally, the completely soluble chitosan solution was titrated with $0.1 \mathrm{M}$ sodium hydroxide solution. The following formula was used to calculate DD,

$$
\mathrm{DD}(\%)=2.03 \cdot \frac{\mathrm{V}_{2}-\mathrm{V}_{1}}{m+0.0042\left(\mathrm{~V}_{2}-V_{1}\right)}
$$

where $\mathrm{m}$ is the weight of sample, $\mathrm{V}_{1}, \mathrm{~V}_{2}$ are the volumes of $0.1 \mathrm{M}$ sodium hydroxide solution corresponding to the deflection points, the number 2.03 is the coefficient resulting from the molecular weight of chitin monomer unit and the number 0.0042 is the coefficient resulting from the difference between molecular weights of chitin and chitosan monomer units [5]. 


\subsection{Preparation of stearoyl chitosan}

Two different degree substituted stearoyl chitosan compounds (DS 79\%, and DS 86\%) were prepared by the method described by Tien et al. [15]. DS 79\% stearoyl chitosan was prepared by starting chitosan (1 g) was dissolved in aqueous acetic acid $(240 \mathrm{~mL}, 0.453 \mathrm{M})$ by stirring for 24 hours. By slow addition of $0.1 \mathrm{M} \mathrm{NaOH}$ with strong agitation, $\mathrm{pH}$ was adjusted to 7.2, yielding gel slurry, the volume of which was adjusted to about 300 $\mathrm{mL}$. Then stearoyl chloride $(5.44 \mathrm{~g}$ ) was added and the reaction volume diluted to $400 \mathrm{~mL}$ with distilled water.

After 7 hours of reaction time, preparation was neutralized ( $\mathrm{pH}$ 6.8-7.0) and precipitated with acetone. The filtered precipitate was washed at $55^{\circ} \mathrm{C}$ with an excess methanol and decanted. The washing was repeated three times to remove fatty acids. Finally, the product was dried with pure acetone to obtain the corresponding derivative powder of DS 79\% stearoyl chitosan compound.

DS $86 \%$ stearoyl chitosan was prepared by starting deacetylated chitosan $(1 \mathrm{~g})$, which was dissolved in aqueous acetic acid $(240 \mathrm{~mL}, 0.453 \mathrm{M})$ by stirring for 24 hours. The rest of the procedure was exactly the same as the method followed to synthesize DS $79 \%$ stearoyl chitosan.

\subsection{Characterization}

FT-IR analysis was carried out on a Nicolet 6700 FT-IR spectrometer (Thermo Electron Co., WI, USA). The resolution was set at $4 \mathrm{~cm}^{-1}$ with a total of 32 scans and the wave number range between 400 and $4000 \mathrm{~cm}^{-1}$. The concentration of $2 \%(\mathrm{w} / \mathrm{v})$ in d-trifluoroacetic acid was used to collect ${ }^{1} \mathrm{H}$ NMR spectrum of stearoyl chitosan on Bruker AV600 spectrometer under a static magnetic field of $600 \mathrm{MHz}$. Data analysis was carried out using the Mnova NMR software.

\subsection{Determination of DS}

The DS of prepared stearoyl chitosan products were calculated by FT-IR spectroscopy [16].

\subsection{Thermogravimetric analysis (TGA)}

The thermal gravimetric analysis of stearoyl chitosan was carried out using a TGA/DTA High Temperature 115 thermogravimetric/differential analyzer (Pyris-Diamond, PerkinElmer, MA, USA). The constant heating rate was set to $10{ }^{\circ} \mathrm{C} / \mathrm{min}$ while the temperature range set to $50{ }^{\circ} \mathrm{C}$ to $450{ }^{\circ} \mathrm{C}$. Analysis was performed under argon atmosphere.

\subsection{X-ray diffraction (XRD)}

XRD analysis was carried out using an X-ray diffractometer (X'Pert Pro MPD, PANalytical B.V., The Netherlands), operating at $40 \mathrm{kV}$ and $20 \mathrm{~mA}$ with a $\mathrm{Cu}-\mathrm{K} \alpha$ source. The diffraction intensity was measured in the range of $2 \theta$ angles between $5^{\circ}$ and $40^{\circ}$, with a step size of $0.020^{\circ}$, step time of $40 \mathrm{~s}$ and scanning speed of $0.0005^{\circ}$ $\mathrm{s}^{-1}$.

\subsection{Solubility studies}

Solubility studies were conducted to find suitable solvents to dissolve stearoyl chitosan compounds with high viscosity, which can be used in various applications. In this study, stearoyl chitosan with DS $86 \%$ was tried to dissolve in the concentration of $2 \%(\mathrm{w} / \mathrm{v})$ in a series of solvents such as isopropanol, trifluoroacetic acid, dichloromethane, dimethylformamide, hexane, toluene, tetrahydrofuran, acetone, chloroform, pyridine, dimethylsulfoxide, trifluoroethanol, dimethylacetamide, and hexafluoroacetone sesquihydrate by sonicating for seven hours $[2,17-20]$.

\subsection{Rheological behavior}

Rheological measurements were performed on a rheometer (DHR2, TA Instruments, DE, USA). The parallel plates with $40 \mathrm{~mm}$ diameter were used to collect data while the plate gap was set to $1000 \mu \mathrm{m}$. Data analysis was carried out using TRIOS_v3.0.2.3156 software.

The frequency studies were conducted for stearoyl chitosan solutions. The solution viscosity of stearoyl chitosan solutions was measured using flow sweep, where the shear rate was linearly increased from 10 to $200 \mathrm{~s}^{-}$ ${ }^{1}$ at $25.0^{\circ} \mathrm{C}$. At there, an equilibration time of $20.0 \mathrm{~s}$ and an averaging time of $10 \mathrm{~s}$ were maintained [2].

\subsection{Determination of DS}

\section{Results and Discussion}

The DS of the prepared stearoyl chitosan products (stearoyl chitosan starting from chitosan and stearoyl chitosan starting from deacetylated chitosan) were calculated by FT-IR spectroscopy [15]. According to the calculated values, the stearoyl chitosan starting from chitosan had DS 79\% while stearoyl chitosan starting from deacetylated chitosan had DS $86 \%$. 


\subsection{Structure characterization}

\subsubsection{FT-IR data analysis}

As shown in Fig.1, the broad band at $3330 \mathrm{~cm}^{-1}$ in chitosan due to inter- and intra- molecular hydrogen bonding of $-\mathrm{NH}_{2}$ and $-\mathrm{OH}$ stretching vibrations was decreased in intensity in stearoyl chitosan derivatives. The two new peaks at 1720 and $1710 \mathrm{~cm}^{-1}$ were due to $\mathrm{C}=\mathrm{O}$ of $-\mathrm{NCOR}$ group and $\mathrm{C}=\mathrm{O}$ of $-\mathrm{OCOR}$ group of stearoyl chitosan, which were resulted from the acylation reaction between stearoyl chloride and $-\mathrm{NH}_{2}$ and $-\mathrm{OH}$ groups of chitosan $[15,21]$. Presence of both 1720 and $1700 \mathrm{~cm}^{-1}$ peaks confirmed that the substitution took place at both $-\mathrm{NH}_{2}$ and $-\mathrm{OH}$ groups in stearoyl chitosan starting from chitosan. Mostly N-substitution took place

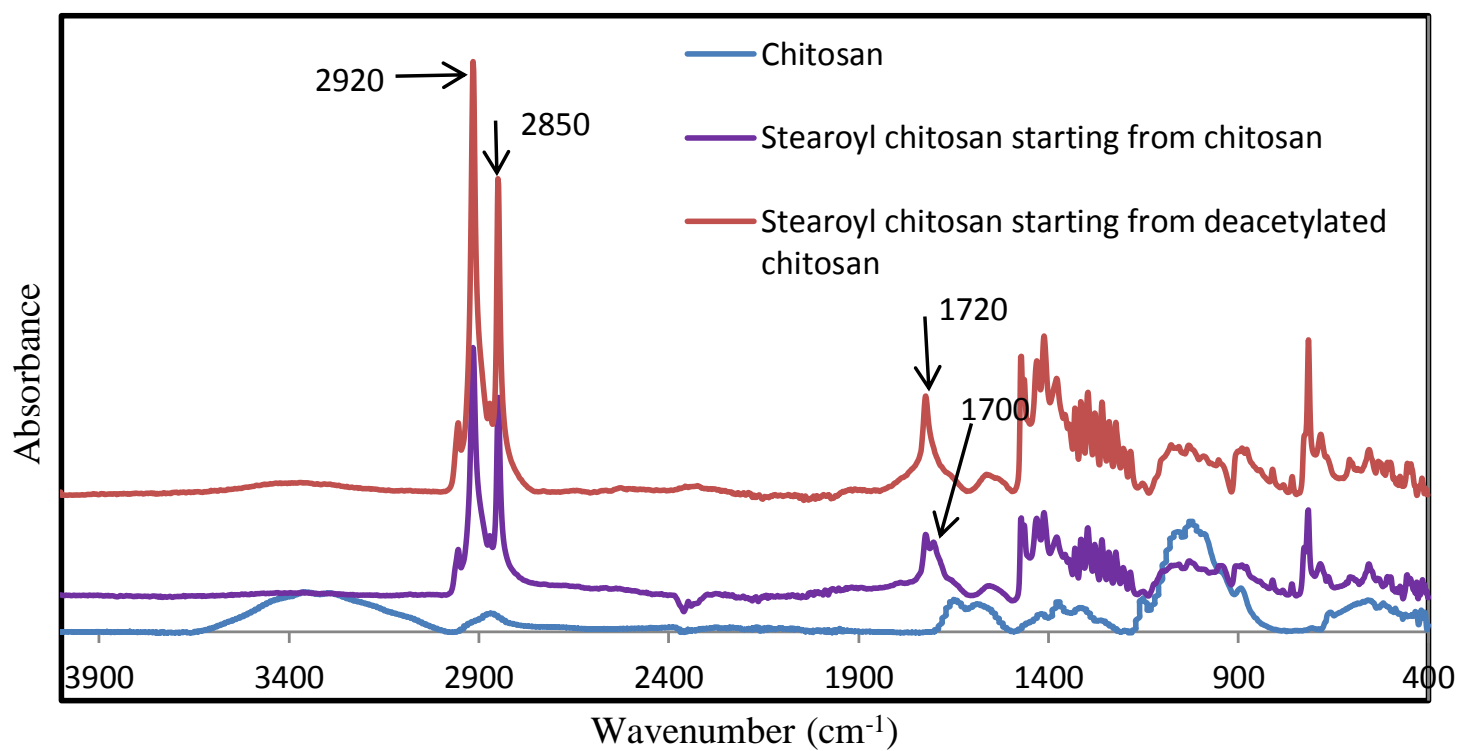

Figure 1: FT-IR spectra of chitosan and stearoyl chitosan.

in stearoyl chitosan starting from deacetylated chitosan due to presence only the intense peak at $1720 \mathrm{~cm}^{-1}$. The peak intensity of $1720 \mathrm{~cm}^{-1}$ was higher in stearoyl chitosan starting from deacetylated chitosan than that in stearoyl chitosan starting from chitosan. This observation was confirmed that more substitution took place in stearoyl chitosan starting from deacetylated chitosan, which has more $\mathrm{N}$ - active sites for acylation, and the activeness of amino group is stronger than that of hydroxyl group, the priority of $-\mathrm{NH}_{2}$ group convert to NCOR was higher than converting $-\mathrm{OH}$ to $-\mathrm{OCOR}$.

The other two prominent peaks around 2920 and $2850 \mathrm{~cm}^{-1}$ were due to asymmetrical and symmetrical bending vibrations of methylene groups of long alkyl chain of stearoyl chitosan [15]. The absorption intensity was higher in stearoyl chitosan starting from deacetylated chitosan than that in stearoyl chitosan starting from chitosan. This observation was confirmed that more substitution took place in stearoyl chitosan starting from deacetylated chitosan. All of these observations were clearly confirmed that the formation of stearoyl chitosan while more substitution took place in stearoyl chitosan starting from deacetylated chitosan.

\subsection{2 ${ }^{1} \mathrm{H}$ NMR data analysis}

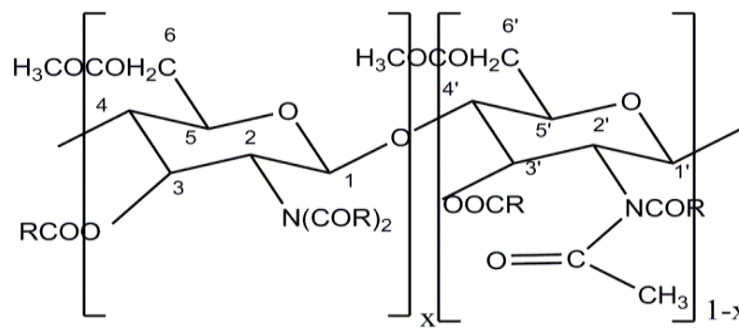

$$
\mathrm{R}=\stackrel{\mathrm{a}}{\mathrm{CH}} \mathrm{CH}_{2}\left(\mathrm{CH}_{2}\right)_{15} \mathrm{CH}_{3}
$$

Figure 2: The protons corresponding to Fig. 3 


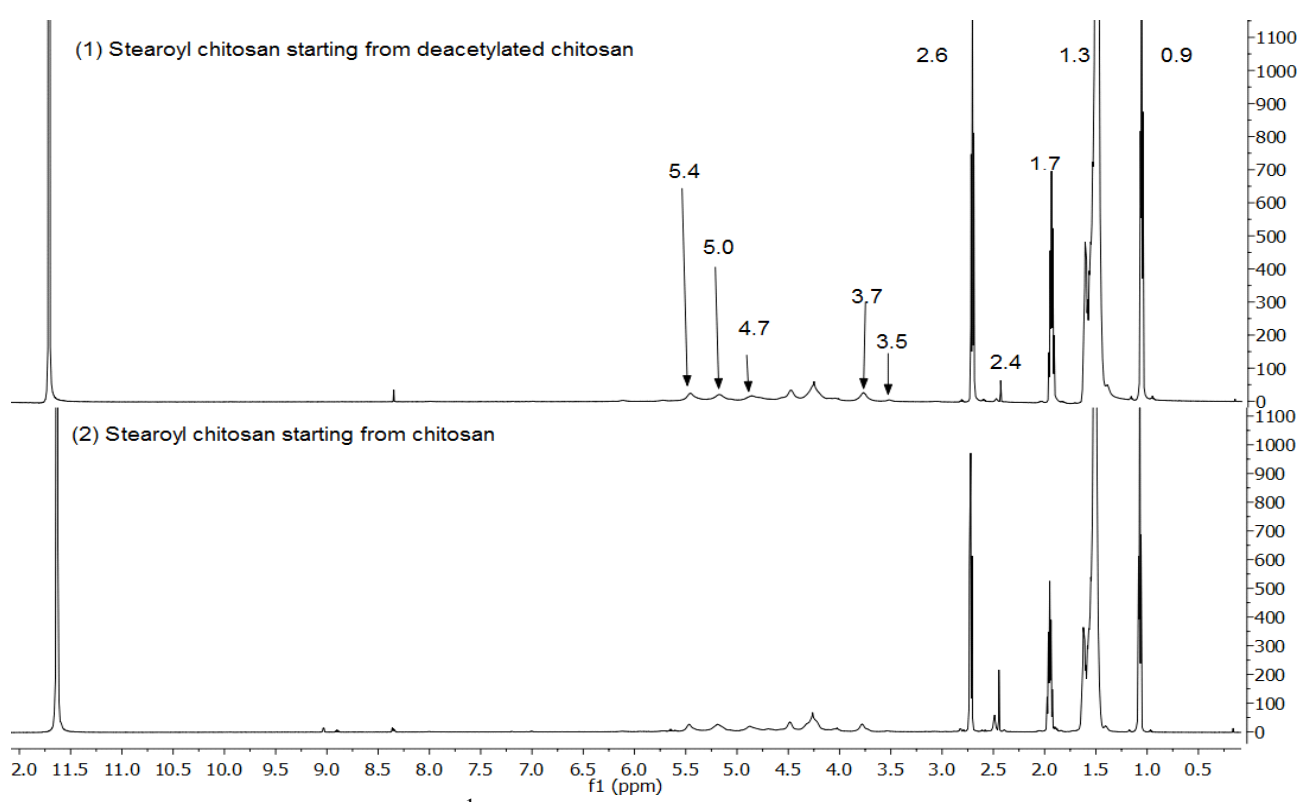

Figure 3: ${ }^{1} \mathrm{H}$ NMR spectra of stearoyl chitosan.

The Fig. 3 illustrated the ${ }^{1} \mathrm{H}$ NMR spectrum of stearoyl chitosan in deuterated trifluoroacetic acid (the dTFA solvent resonance is at $11.5 \mathrm{ppm}$ ). The protons corresponding to Fig. 2 are shown in Fig. 3 [19]. As shown in Fig. 3, the resonances formed at 5.4, 5.0, $4.7 \mathrm{ppm}$ were attributed to $\mathrm{H} 1, \mathrm{H} 3$ and $\mathrm{H} 4$ protons of chitosan, respectively. The resonances were at 3.5 and $3.7 \mathrm{ppm}$ due to $\mathrm{H} 6$ and $\mathrm{H} 5$ of the ring protons (Scheme 5.7). The ${ }^{1} \mathrm{H}$ NMR data of Ma et al. [19] was confirmed by the presence of a multiplet peak at $2.6 \mathrm{ppm}$ and the resonance at $2.4 \mathrm{ppm}$ attributed to Ha. The broad multiplet peaks from 1.3 to $1.7 \mathrm{ppm}$ were due to $-\mathrm{CH}_{2}$ groups of stearoyl chloride chain while a typical resonance at $0.9 \mathrm{ppm}$ was due to $-\mathrm{CH}_{3}$ of stearoyl group.

\subsection{Thermogravimetric analysis (TGA)}

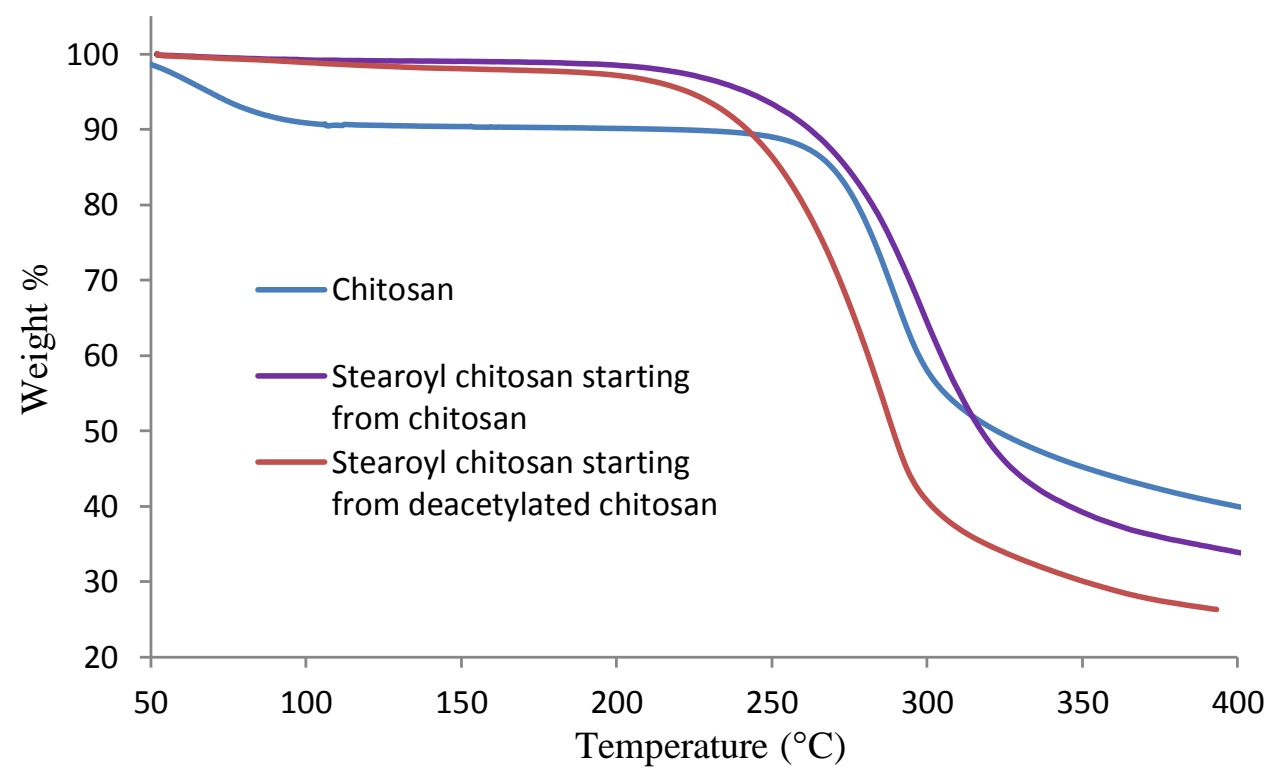

Figure 4: TGA curves for chitosan and stearoyl chitosan.

As shown in Fig. 4, the onset of degradation occurred at $251.5{ }^{\circ} \mathrm{C}, 242.24{ }^{\circ} \mathrm{C}$ and $226.6{ }^{\circ} \mathrm{C}$ for native chitosan, stearoyl chitosan starting from chitosan and stearoyl chitosan starting from deacetylated chitosan, respectively. Therefore, the thermal stability of stearoyl chitosan was lower than that of parent chitosan. The thermal stability of stearoyl chitosan starting from chitosan (DS 79\%) was higher than that of stearoyl chitosan starting from deacetylated chitosan (DS 86\%). Hence, the thermal stability of stearoyl chitosan decreased with increased DS. This could be due to less number of hydrogen bond formations in highly substituted stearoyl chitosan [22]. 


\subsection{X-ray diffraction (XRD)}

In Fig. 5, there was an intense peak around $20^{\circ}$ in chitosan while new sharp peaks were observed between $20^{\circ}$ and $25^{\circ}$ in stearoyl chitosan. Among the two stearoyl chitosan products, stearoyl chitosan starting from deacetylated chitosan which has a DS of $86 \%$ showed the highest peak intensity. This suggested that stearoyl chitosan had more crystalline and possibly more stable organization than that of chitosan [15].

The calculated DO values for chitosan, stearoyl chitosan starting from chitosan (DS 79\%) and stearoyl chitosan starting from deacetylated chitosan (DS 86\%) were 398 counts $\left({ }^{\circ}\right)^{-1}, 1772$ counts $\left({ }^{\circ}\right)^{-1}$ and 8797 counts $\left({ }^{\circ}\right)^{-1}$, respectively. Therefore, stearoyl chitosan had a higher DO than chitosan. The DO of stearoyl chitosan increased with increased DS. Hence, stearoyl chitosan starting from deacetylated chitosan had more crystalline and stable organization.

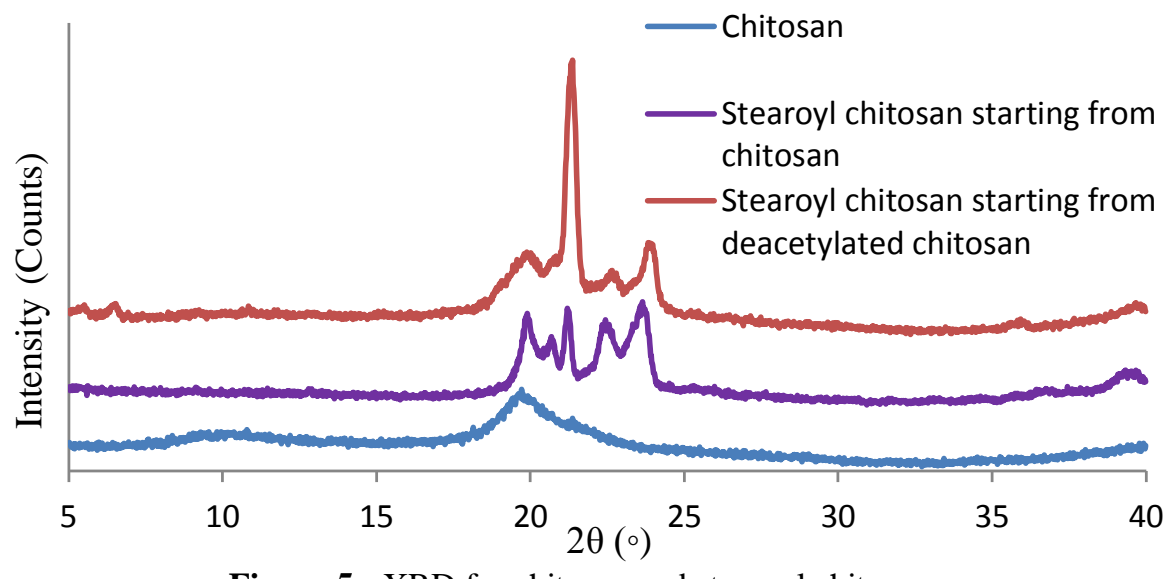

Figure 5: XRD for chitosan and stearoyl chitosan.

\subsection{Solubility studies and rheological behavior}

According to the solubility studies, toluene and pyridine were the best solvents to dissolve stearoyl chitosan with DS $86 \%$, which had the highest degree of substitution. But, stearoyl chitosan was dissolved only up to $2 \%$ concentration in toluene and pyridine. Therefore, the rheological properties were assessed in $2 \%(\mathrm{w} / \mathrm{v})$ stearoyl chitosan in toluene and pyridine.

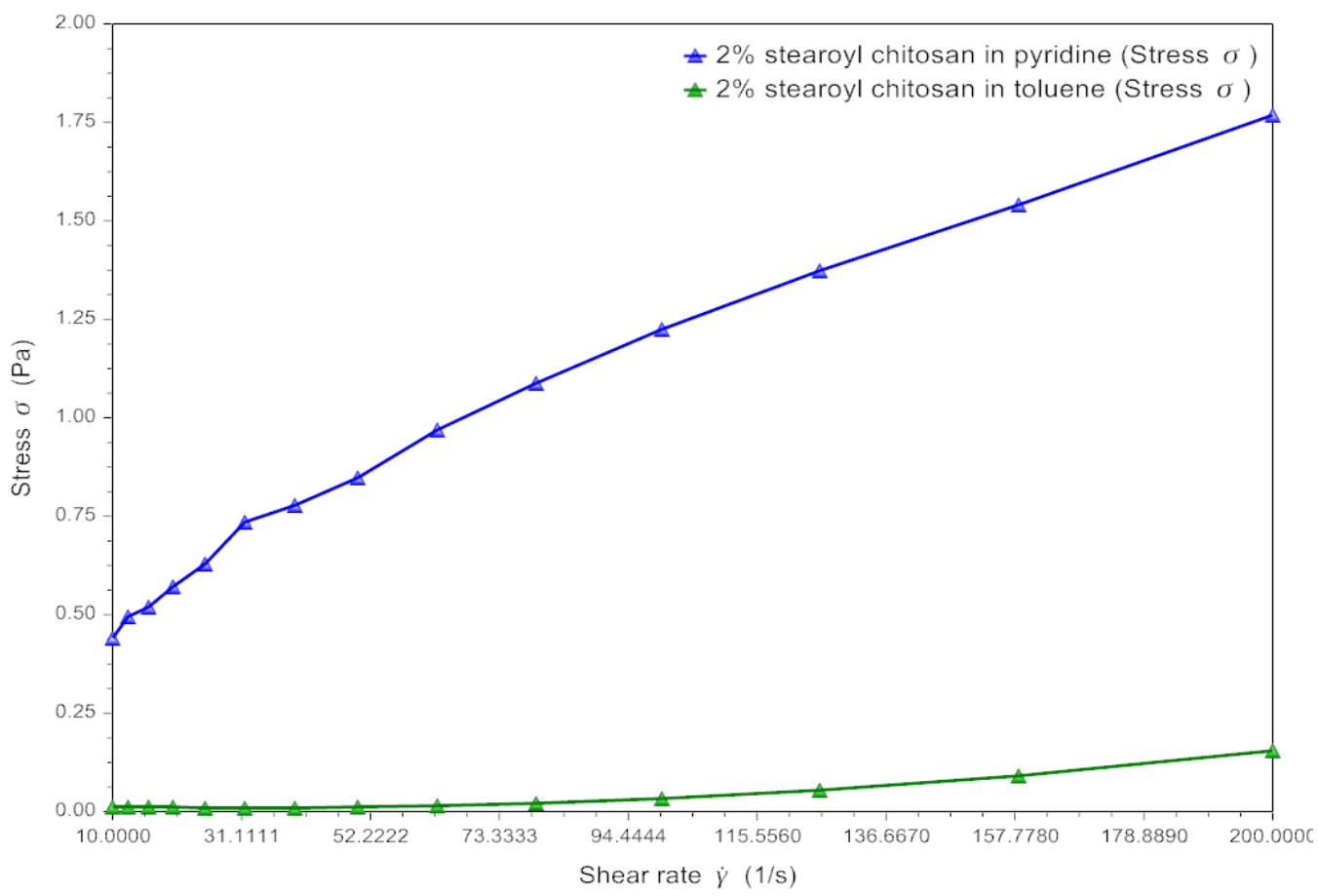

Figure 6: Influence of shear rate and different solvents on shear stress of $2 \%$ stearoyl chitosan solutions.

In fig. 6, the shear stress of stearoyl chitosan in pyridine and toluene did not increase in direct proportion to shear rate while the deviation was in the direction of shear thinning. Therefore, stearoyl chitosan dissolved in 
pyridine and stearoyl chitosan dissolved in toluene exhibited shear thinning behavior. The shear stress value was higher for stearoyl chitosan dissolved in pyridine than toluene at the same shear rate. This could be due to the increased viscosity of pyridine, which is $0.88 \mathrm{cP}$ compared to the viscosity of toluene, which is $0.59 \mathrm{cP}$. Also it may be due to the formation of strong intermolecular interactions in stearoyl chitosan dissolved in pyridine than that in toluene.

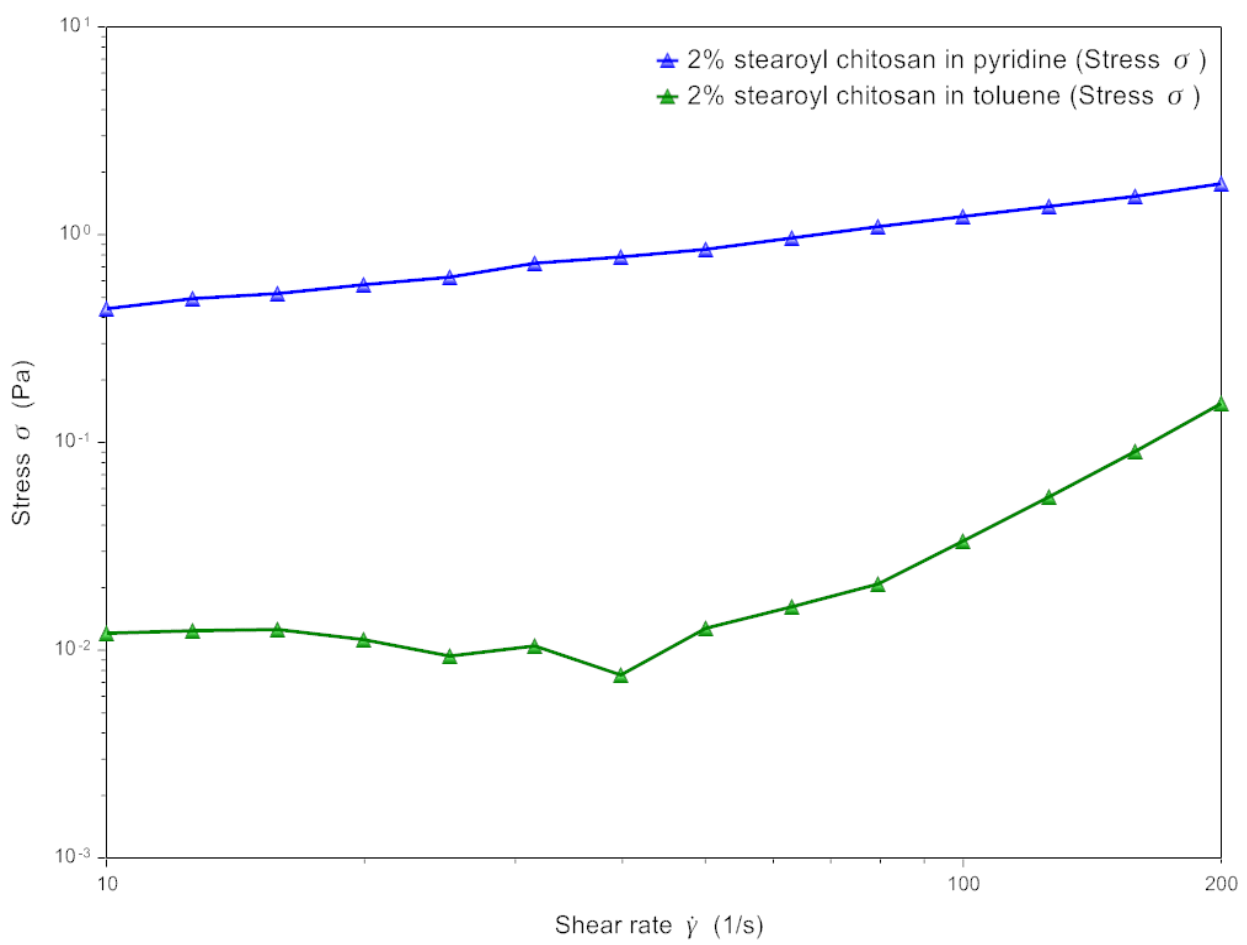

Figure 7: Power law plots (log shear stress versus log shear rate) of stearoyl chitosan solutions.

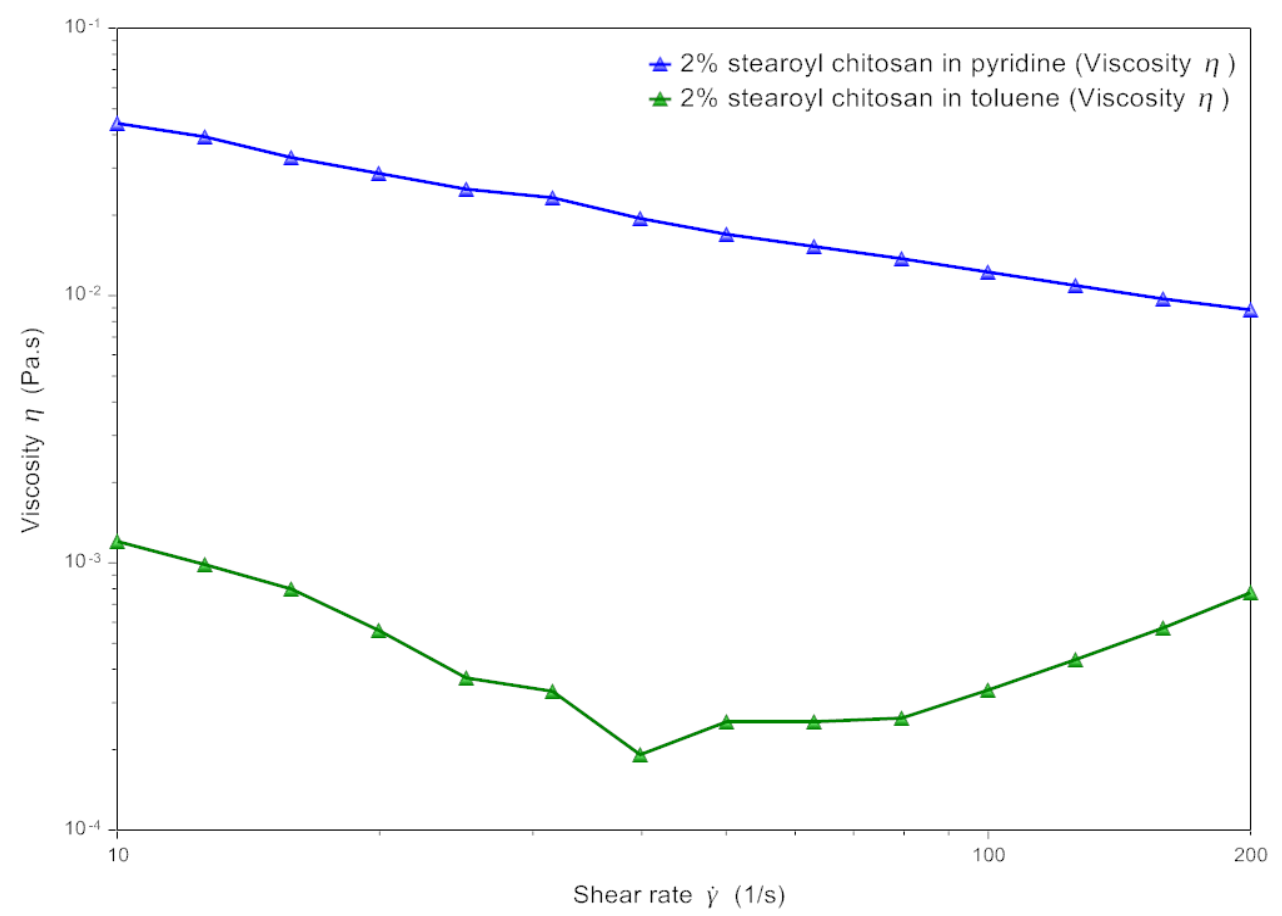

Figure 8: Viscosity as a function of shear rate for stearoyl chitosan dissolved in pyridine and in toluene.

As shown in fig.6, the slopes of two plots were $<1$. Therefore, stearoyl chitosan in both pyridine and toluene showed shear thinning behavior. The viscosities of stearoyl chitosan in pyridine and stearoyl chitosan in toluene were decreased with increased shear rate (Fig. 8). Also the viscosities at shear rate $10 \mathrm{~s}^{-1}$ for $2 \%$ stearoyl chitosan solutions were 0.044 Pa.s and 0.0012 Pa.s for in pyridine and in toluene solvents, respectively. Since 
the conditions were maintained same for two solutions of stearoyl chitosan, the higher solution viscosity for stearoyl chitosan in pyridine was due to higher viscosity of pyridine than that of toluene.

\section{Conclusion}

Two different degree substituted stearoyl chitosan compounds were synthesized as DS $79 \%$ and DS $86 \%$. According to TGA data, the thermal stability of stearoyl chitosan was lower than that of parent chitosan. Also the thermal stability of stearoyl chitosan decreased with increased DS. According to XRD data, crystallinity of stearoyl chitosan increased with increased DS while stearoyl chitosan had stable organization than chitosan. Solubility studies showed that the suitable solvents to dissolve stearoyl chitosan with DS $86 \%$ are toluene and pyridine but only up to $2 \%(\mathrm{w} / \mathrm{v})$ concentration. The $2 \%(\mathrm{w} / \mathrm{v})$ stearoyl chitosan (DS $86 \%$ ) solutions displayed shear thinning behavior. The viscosities at shear rate $10 \mathrm{~s}^{-1}$ for $2 \%$ stearoyl chitosan solutions were 0.044 Pa.s and 0.0012 Pa.s for in pyridine and in toluene solvents, respectively. Since $2 \%$ stearoyl chitosan dissolved in pyridine had high viscosity with shear thinning property, it can be suitable as rheology modifiers.

\section{Acknowledgement}

Authors would like to thank Dr. Andrew Goudy and Dr. Noureddine Melikechi of Delaware State University and Dr. Steve Bai, NMR Laboratory of University of Delaware, DE, USA.

\section{References}

[1] E.A. El-hefian, and A.H. Yahaya, Rheological study of chitosan and its blends: An overview. Maejo Internaional Journal of Science and Technology, 4(2), 2010, 210-220.

[2] P. Katugampola, and C. Winstead, Rheological behavior and thermal stability of palmitoyl chitosan varying the degree of substitution. International Journal of Pharmaceutical Science Invention, 3(11), 2014, 24-32.

[3] M.R. Radrigues, Synthesis and investigation of chitosan derivatives formed by reaction with acyl chlorides. Journal of Carbohydrate Chemistry, 24(1), 2005, 41-54.

[4] I. Aranaz, M. Mengibar, R. Harris, I. Panos, B. Miralles, N. Acosta, G. Galed, and A. Heras, Functional characterization of chitin and chitosan. Current Chemical Biology, 3(2), 2009, 203-230.

[5] R. Czechowska-Biskup, D. Jarosinska, B. Rokita, P. Ulanski, and J.M. Rosiak, Determination of degree of deacetylation of chitosancomparison methods. Progress on Chemistry and Application of Chitin and its Derivatives, 17, 2012, 5-20.

[6] T. A. Khan, K.K. Peh, and H.S. Ch'ng, Reporting degree of deacetylation values of chitosan: the influence of analytical methods. Journal of Pharmacy and Pharmaceutical Sciences, 5(3), 2002, 205-212.

[7] D. Yanming, X. Congyi, W. Jianwei, W. Mian, W. Yusong, and R. Yonghong, Determination of degree of substitution for N-acylated chitosan using IR spectra. Science in China Series B: Chemistry, 44(2), 2000, 216-224.

[8] C. Winstead, and P. Katugampola, Rheological and structural studies of carboxymethyl derivatives of chitosan, AIP conference proceedings, 1599(1), 2014, 290-293.

[9] M.P. Stevens, Polymer Chemistry; An introduction (New York: Oxford University Press, 2011)

[10] D. Zhang, A.B. Karki, D. Rutman, D.P. Young, A. Wang, D. Cocke, T.H. Ho, and Z. Guo, Electrospun polyacrylonitrile nanocomposites fibers reinforced with $\mathrm{Fe}_{3} \mathrm{O}_{4}$ nanoparticles: fabrication and property analysis, Polymer, 50(17), $2009,4189-4198$.

[11] R. Mucha, Rheological characteristics of semi-dilute chitosan solutions, Macromolecular Chemistry and Physics, 198(2), 1997, 471484.

[12] Y. Yuan, B.M. Chesnutt, W.O. Haggard, and J.D. Bumgardner, Deacetylation of chitosan: Material characterization and in vitro evaluation via albumin absorption and pre-osteoblastic cell cultures, Materials, 4(8), 2011, 1399-1416.

[13] Z.T. Zhang, D.H. Chen, and L. Chen, Preparation of two different serials of chitosan, Journal of Dong Hua University (English Edition), 19(3), 2002, 36-39.

[14] Y. Dong, C. Xu, J. Wang, M. Wang, Y. Wu, and Y. Ruan, Determination of degree of substitution for N-acylated chitosan using IR spectra, Science in China. Series B: Chemistry, 44(2), 2001, 216-224.

[15] C.L. Tien, M. Lacroix, P. Ispas-Szabo, and M. Mateescu, N-acylated chitosan: hydrophobic matrices for controlled drug release, Journal of Controlled Release, 93(1), 2003, 1-13.

[16] Z. Li, X. Zhuang, X. Liu, Y. Guan, and K. Yao, Rheological study on O-carboxymethylated chitosan/cellulose polyblends from LiCl/N, N-dimethylacetamide solution, Journal of Applied Polymer Science, 88(7), 2003, 1719-1725.

[17] C.K.S. Pillai, W. Paul, and C.P. Sharma, Chitin and chitosan polymers: Chemistry, solubility and fiber formation, Progress in Polymer Science, 34(7), 2009, 641-678.

[18] M.R. Radrigues, Synthesis and investigation of chitosan derivatives formed by reaction with acyl chloride, Journal of Carbohydrate Chemistry, 24(1), 2005, 41-54.

[19] G. Ma, D. Yang, J.F. Kennedy, and J. Nie, Synthesize and characterization of organic-soluble acylated chitosan, Carbohydrate Polymers, 75(3), 2009, 390-394.

[20] S. Fujii, H. Kumagai, and M. Noda, Preparation of poly(acyl)-chitosans. Carbohydrate Research, 83, 1980, $389-393$.

[21] Y.W. Cho, J. Jang, C.R. Park, and S.W. Ko, Preparation and solubility in acid and water of partially deacetylated chitins, Biomacromolecules, 1(4), 2000, 609-614.

[22] A.A.Fahd, and I. Tirkistani, Thermal analysis of some chitosan Schiff bases, Polymer Degradation and Stability, 60(1), 1998, 67-70. 